\title{
Neutrino electromagnetic form factors
}

\author{
P. Vogel and J. Engel \\ Physics Department, California Institute of Technology, Pasadena, California 91125
}

(Received 27 December 1988)

\begin{abstract}
It has been suggested that an apparent correlation of the flux of detected solar neutrinos with solar activity is due to a neutrino magnetic moment. Here several terrestrial experiments that might observe the magnetic moment are considered, with emphasis on those employing reactor neutrinos. The neutrino charge radius, and prospects for observing it, are also discussed. An appendix collects all relevant neutrino scattering cross sections.
\end{abstract}

\section{INTRODUCTION}

The flux of solar neutrinos detected on Earth ${ }^{1}$ appears to be anticorrelated with sunspot activity. Voloshin, Vysotskii, and $\mathrm{Okun}^{2}$ pointed out that such an effect would follow from an electron-neutrino magnetic dipole moment of magnitude

$$
\mu_{v_{e}} \approx 10^{-10},
$$

where, as everywhere else in our paper, we use units of electron Bohr magnetons $e / 2 m_{e}$. A significant number of left-handed neutrinos with magnetic moment (1) would be flipped into sterile right-handed neutrinos by the magnetic field in the solar convective zone. Since the strength of this field presumably follows solar activity, the number of left-handed neutrinos detected on Earth would reach a minimum during maximum activity and vice versa.

The suggestion that this in fact occurs has stimulated a reexamination of the question of neutrino magnetic moments. In the minimal standard electroweak model, neutrinos are massless and have no magnetic moment. A simple extension permits massive Dirac neutrinos to acquire through radiative corrections ${ }^{3}$ a moment

$$
\mu_{v}=\frac{3 G_{F} m_{e} m_{v}}{4 \sqrt{2} \pi^{2}}=3.2 \times 10^{-19}\left(\frac{m_{v}}{1 \mathrm{eV}}\right)
$$

that is nonzero but too small to affect astrophysical phenomena. The incorporation of additional physics, e.g., right-handed currents, leads to much larger magnetic moments ${ }^{4}\left[m_{v}\right.$ in (2) is replaced by $m_{l} \sin 2 \xi$, where $\xi$ is the mixing angle connecting the mass and weak-interaction eigenstates of the two intermediate vector bosons $W$ and $m_{l}$ is the charged-lepton mass], but even this enhancement is still far from that required to explain the solarneutrino deficit. Scenarios that do allow magnetic moments as large as (1) for light $(\sim \mathrm{eV})$ neutrinos ${ }^{5}$ deviate significantly from the standard model but have been proposed. In many cases the models consider not only diagonal magnetic moments, but also nondiagonal transition moments which, under the effect of a transverse magnetic field, transform left-handed neutrinos of one flavor into right-handed neutrinos (or antineutrinos) of another flavor. Only massive Dirac particles can have nonvanishing diagonal magnetic moments. No such restriction applies to transition moments, however, and both Dirac and Majorana neutrinos may have them. In either case, at least four neutrino components (e.g., a massive Dirac particle or a pair of Majorana particles) couple to the same charged lepton.

In this paper, we will analyze the feasibility of neutrino-lepton scattering experiments to measure neutrino magnetic moments and charge radii, with emphasis on experiments at reactor energies. Because the recoiling lepton rather than the scattered neutrino is observed, a flavor-changing moment cannot be distinguished from a diagonal moment. Although the discussion here is framed in terms of Dirac neutrinos with diagonal moments, our results apply as well to transition moments (including the magnetic-electric dipole interference ${ }^{6}$ ), and to Majorana neutrinos if the charge radius is replaced by an anapole moment. ${ }^{7}$

\section{EXISTING LIMITS}

A variety of astrophysical and cosmological arguments restrict the magnitude of the neutrino magnetic moment. For example, ${ }^{4} \mathrm{He}$ nucleosynthesis ${ }^{8}$ allows at most one flavor to have $\mu_{v}>5 \times 10^{-11}$, and stellar cooling requires 9 $\mu_{v_{e}} \leq 1 \times 10^{-11}$. As recognized by a large number of authors, the observation of neutrinos from SN 1987A can be interpreted as a further restriction. Left-handed neutrinos (with moments) produced during the collapse would flip their helicity in collisions with the electrons and nuclei of the collapsing star. The resulting righthanded neutrinos would in the simplest scenario be sterile with respect to the weak interaction, and therefore escape, reducing the trapped lepton number of the star and impeding the progress of the supernova. In addition, the escaping right-handed neutrinos, with average energy $\approx 100 \mathrm{MeV}$, could be reflipped by intergalactic magnetic fields, and detected with relatively high efficiency on Earth. The absence of such a signal from SN 1987A is used to place the limits $\mu_{v} \leq 5 \times 10^{-13}$ in Ref. 10, $\mu_{v} \leq(1-10) \times 10^{-13}$ in Ref. 11 , and $\mu_{v} \leq 2 \times 10^{-12}$ in Ref. 12. These values, although not quite consistent with one another, seem to exclude the moment in Eq. (1) by a fac- 
tor of 100 or more. However, the analysis clearly depends on, among other things, the assumption that right-handed neutrinos are in fact able to escape the star. A direct measurement of the neutrino magnetic moment would be of great use.

Some relevant laboratory data do exist. Neutrinoelectron scattering can be used to set limits on the value of $\mu_{v}$ if the measured cross section is compared with Eq. (A5). For muon neutrinos, the limit is ${ }^{13} \mu_{v_{\mu}} \leq 10^{-9}$. For electron neutrinos two experiments are available. Reference 14 reports results with $v_{e}, v_{\mu}$, and $\bar{v}_{\mu}$ from the LAMPF beam stop which, assuming $\sin ^{2} \theta_{W}=0.226$, yield a limit of $\approx 1.3 \times 10^{-9}$ to $\mu_{v_{e}}$. In a much earlier experiment, the first of its kind, Reines, Gurr, and Sobel ${ }^{15}$ observed scattering of reactor $\bar{v}_{e}$ on electrons. Their result is interpreted by Kyudliev ${ }^{16}$ as an upper limit of $1.5 \times 10^{-10}$ on the magnetic moment $\mu_{\bar{v}_{e}}$. We believe that the conclusion is not quite justified. The cross sections of Ref. 15, integrated over the reactor antineutrino spectrum, were $(7.6 \pm 2.2) \times 10^{-46} \mathrm{~cm}^{2}$ for electron recoil in the interval $1.5-3.0 \mathrm{MeV}$, and $(1.9 \pm 0.5) \times 10^{-46} \mathrm{~cm}^{2}$ for energies between 3.0 and $4.5 \mathrm{MeV}$. When compared with the now well-understood reactor $\bar{v}_{e}$ spectrum, which we discuss shortly, and the presently accepted value of $\sin ^{2} \theta_{W}$, these cross sections are $1.35 \pm 0.4$ and $2.0 \pm 0.5$ times larger than expected. (The exact analysis is difficult, because the detection efficiency probably depended on the recoil energy; no attempt was made to include that effect here.) This slight discrepancy taken literally would imply a neutrino magnetic moment on the order of $(2-4) \times 10^{-10}$. At the very least, we must conclude that all currently existing direct experimental limits are substantially larger than the value in Eq. (1). A better experiment would definitely bear on the question of magnetic moments and solar neutrinos. In the next section we discuss measurements with low-energy neutrinos to see how much better they can be expected to do.

\section{MAGNETIC MOMENTS AND REACTOR EXPERIMENTS}

In the Appendix we collect all relevant formulas for the scattering cross sections that depend on the neutrino magnetic moment. The scattering on nucleons or nuclei is important in astrophysical applications; in laboratory experiments, however, detection of the very low recoil of baryonic targets appears to be impossible with present technology. Electrons are clearly the most promising targets. The total neutrino-electron cross section increases linearly with $E_{v}$ for $Z$ and $W$ exchange but only logarithmically for the magnetic moment interaction. The relative strength of magnetic scattering is, therefore, highest when $E_{v}$ is small. Furthermore, the $1 / T$ dependence of the magnetic differential cross section [see (A5)] shows that the detection of low recoil is crucial to a good measurement.

All these considerations point to nuclear reactors as the most convenient neutrino sources. Power reactors emit large numbers of antineutrinos, about $5 \times 10^{20} \bar{v}_{e}$ per second, broadly distributed over energies up to about 10
TABLE I. Fit to $\bar{v}_{e}$ spectra above $2 \mathrm{MeV}$ [resulting spectrum in units of $\bar{v}_{e} /(\mathrm{MeV}$ fission); see Eq. (4) for a definition of the variables $a_{i}$ ].

\begin{tabular}{cccccc}
\hline$a_{i}$ & ${ }^{235} \mathrm{U}$ & ${ }^{239} \mathrm{Pu}$ & ${ }^{238} \mathrm{U}$ & ${ }^{241} \mathrm{Pu}$ & ${ }^{252} \mathrm{Cf}$ \\
\hline$a_{0}$ & 0.870 & 0.896 & 0.976 & 0.793 & 1.044 \\
$a_{1}$ & -0.160 & -0.239 & -0.162 & -0.080 & -0.232 \\
$a_{2}$ & -0.0910 & -0.0981 & -0.0790 & -0.1085 & -0.0982 \\
\hline \hline
\end{tabular}

$\mathrm{MeV}$, with a peak at $0.5-1.0 \mathrm{MeV}$. The relevant quantity for scattering experiments is the folded differential cross section

$$
\left\langle\frac{d \sigma}{d T}\right\rangle=\int_{E_{v}^{\min }(T)}^{\infty} \frac{d N_{v}}{d E_{v}} \frac{d \sigma\left(E_{v}\right)}{d T} d E_{v},
$$

where $T$ is the electron recoil energy, $d N_{v} / d E_{v}$ is the reactor $\bar{v}_{e}$ spectrum and $E_{v}^{\min }(T)$ is obtained from (A4). To interpret data, we, therefore, must have accurate knowledge of the antineutrino spectrum, including its low-energy part. At energies above the threshold of 1.8 $\mathrm{MeV}$ for inverse neutron beta decay, the reactor spectrum is known quite well (see Ref. 17 for a description and references). For practical purposes it can be approximated by the function

$$
d N_{v} / d E_{v}=\exp \left(a_{0}+a_{1} E_{v}+a_{2} E_{v}^{2}\right)
$$

where the fitted parameters for the nuclear fuels of interest are listed in Table I, and the antineutrino energy $E_{v}$ is in MeV. (In Tables I and II the entries for ${ }^{235} \mathrm{U}$, ${ }^{239} \mathrm{Pu}$, and ${ }^{241} \mathrm{Pu}$ are for the thermal neutron fission, ${ }^{238} \mathrm{U}$ is for $0.5-\mathrm{MeV}$ neutron fission, and ${ }^{252} \mathrm{Cf}$ is for spontaneous fission. The tables assume that the fission products have been accumulating for 2 years of "exposure time.") The parameters in Table I do not account for a change of slope in the $\bar{v}_{e}$ spectra that takes place above $8 \mathrm{MeV}$; between 8 and $12 \mathrm{MeV}$ the spectra are overestimated by a factor of 2-3. This deficiency is not very important in practice, however, because very few antineutrinos have those energies.

The spectra at lower energies have never been calculated in a systematic way before. Our results, based on a

TABLE II. Calculated antineutrino spectra in units of $\bar{v}_{e} /(\mathrm{MeV}$ fission).

\begin{tabular}{lccccc}
\hline$E(\mathrm{MeV})$ & ${ }^{235} \mathrm{U}$ & ${ }^{239} \mathrm{Pu}$ & ${ }^{238} \mathrm{U}$ & ${ }^{241} \mathrm{Pu}$ & ${ }^{252} \mathrm{Cf}$ \\
\hline 2.0 & 1.26 & 1.08 & 1.50 & 1.32 & 1.27 \\
1.5 & 1.69 & 1.48 & 1.97 & 1.75 & 1.71 \\
1.0 & 2.41 & 2.32 & 2.75 & 2.63 & 2.66 \\
0.75 & 2.66 & 2.58 & 2.96 & 2.90 & 2.92 \\
0.50 & 2.66 & 2.63 & 2.91 & 2.82 & 2.83 \\
0.25 & 2.16 & 2.08 & 2.18 & 2.14 & 2.37 \\
0.125 & 1.98 & 1.99 & 2.02 & 1.85 & 1.63 \\
$6.25 \times 10^{-2}$ & 0.61 & 0.64 & 0.65 & 0.59 & 0.53 \\
$3.12 \times 10^{-2}$ & 0.35 & 2.13 & 1.32 & 3.00 & 3.30 \\
$1.563 \times 10^{-2}$ & 0.092 & 0.56 & 0.35 & 0.79 & 0.87 \\
$7.813 \times 10^{-3}$ & 0.024 & 0.14 & 0.089 & 0.20 & 0.22 \\
\hline \hline
\end{tabular}


summation of the allowed shape beta decays of all fission fragments, are summarized in Table II and Fig. 1. The striking feature of Fig. 1, apparent also in the table, is the seemingly random discontinuity in the gradually decreasing amplitudes. These steps are quite real; they stem from the Coulomb attraction of the emitted electron and the nucleus. Because of it there is a finite probability of emitting a zero-momentum electron, and hence a maximum-energy antineutrino. Whenever such an endpoint is crossed a step in the sum spectrum results. The positions of the steps are very well defined, but their amplitudes depend on the corresponding branching ratios and on the fission yield, which in turn depends on the exposure time and to some extent on the neutron flux in the reactor (effect of neutron capture on long-lived fission fragments). These quantities, which vary from reactor to rector, can all be correctly included in the calculation. It is the integral of the spectrum that appears in Eq. (3), however, so the details at low energies are not terribly important.

In Fig. 2 we show the folded cross sections for the weak and magnetic interactions separately. The steep decrease at recoil energies above $1 \mathrm{MeV}$ is caused by the corresponding drop in the reactor antineutrino spectra. The necessity of observing relatively low electron recoil is apparent from the figure. In a real experiment, one presumably will measure the number of recoil electrons as a function of $T$ and compare the resulting shape and magnitude with expectation based on the weak interaction alone (because that is calculable provided the reactor antineutrino spectrum is known). Thus the important quantity is the total/weak folded cross-section ratio, shown in Fig. 3 for several values of $\mu_{v}$. Assuming somewhat arbitrarily that $10 \%$ accuracy is achievable, we see that recoil of $4 \mathrm{MeV}$ must be observed in order to be sensitive to $\mu_{v}=10^{-10}$, while one has to go down to $T=200$ $\mathrm{keV}$ for $3 \times 10^{-11}$, and to $25 \mathrm{keV}$ for $10^{-11}$. The experience of Reines, Gurr, and Sobel ${ }^{15}$ indicates that $10^{-10}$ is clearly within reach, $3 \times 10^{-11}$ is difficult but perhaps not impossible, and $10^{-11}$ is almost out of the question. Thus, the range of limits achievable by a reactor experi-

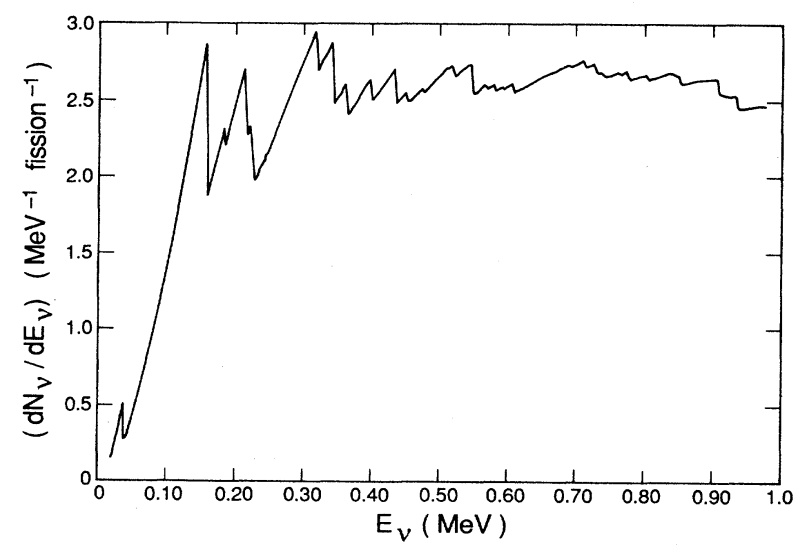

FIG. 1. Low-energy part of the antineutrino spectrum, corresponding to ${ }^{235} \mathrm{U}$ fission. Two years exposure time.

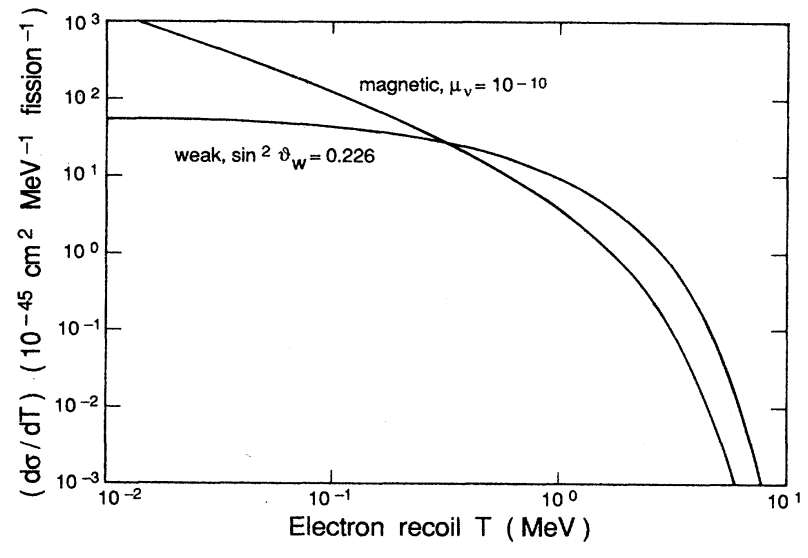

FIG. 2. Differential cross section for $\bar{v}_{e}$ scattering on electrons, averaged over the antineutrino spectrum of fissioning ${ }^{235} \mathrm{U}$.

ment encompasses the value (1) required to explain the solar-neutrino problem, though just barely.

Instead of a nuclear reactor an electron-capture radioactive source could in principle be used. The advantage is a possible $4 \pi$ geometry; i.e., the detector might completely surround the source. The whole apparatus could be placed in an underground laboratory in order to minimize background. To illustrate such an experiment, let us consider the same $600-\mathrm{kCi}{ }^{51} \mathrm{Cr}$ source that is to be used for calibration in the GALLEX project. ${ }^{18}$ (Note that even this very strong source is about $10^{4}$ times weaker than a power reactor.) We assume that at our disposal is a 50-cm-thick liquid-argon detector, and that recoil electrons can be detected from $T_{\min }=50 \mathrm{keV}$; background is neglected. The weak interaction then leads to 5.6 counts per hour in the detector, and the neutrino magnetic moment to $6.1 \times 10^{20} \mu_{v}^{2}$ counts per hour. If the total cross section can be determined to $10 \%$ accuracy, the detection limit on $\mu_{v_{e}}$ is $\approx 3 \times 10^{-11}$. If the differential cross section can be determined to the same

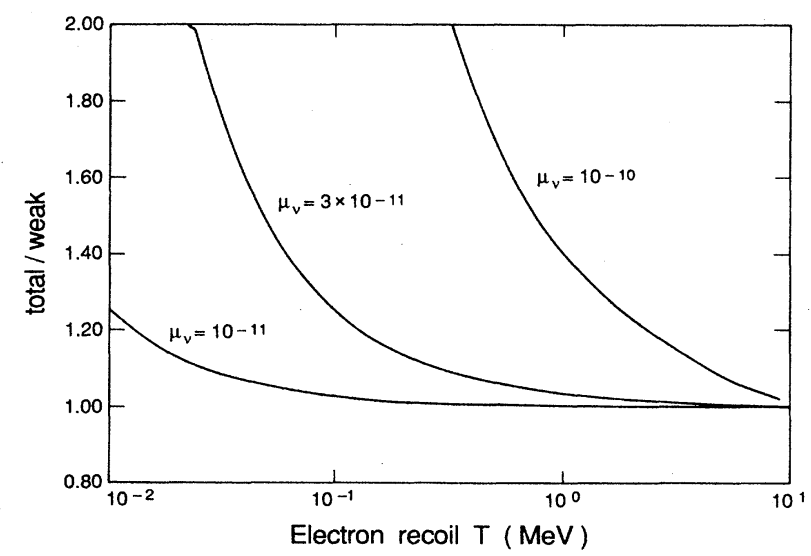

FIG. 3. Ratio of total to weak-interaction cross sections for different values of the neutrino magnetic moment, averaged over the antineutrino spectrum of fissioning ${ }^{235} \mathrm{U}$. 
accuracy the limit is $\mu_{v_{e}} \approx 1.4 \times 10^{-11}$ from the lowest recoil electrons. These values are comparable to the detection limits discussed above for nuclear reactors.

\section{ASYMMETRY IN SOLAR-NEUTRINO SCATTERING}

A neutrino magnetic moment has further consequences in the Sun. While the weak interaction ( $W$ and $Z$ exchange) preserves neutrino helicity, photon exchange involving a neutrino magnetic moment flips it. For longitudinally polarized neutrinos the two interactions do not interfere, and consequently the cross section (A5) depends quadratically on $\mu_{v}$. Barbieri and Fiorentini ${ }^{19}$ pointed out that under special circumstances an interference between weak and magnetic interactions is possible for solar neutrinos, particularly those from the decay of ${ }^{8} \mathbf{B}$. They originate in the inner part of the Sun, and probably all see the same solar magnetic field. Under its action the neutrino spins precess coherently through an angle that is independent of energy. To achieve the nominal reduction of $\frac{1}{3}$ in the left-handed component, the neutrinos must precess an angle $\cos ^{2} \theta / 2=\frac{1}{3}$, leaving them with a large transverse spin component. Scattering of transversely polarized neutrinos is described by Eq. (A9); the recoil electrons will be preferentially distributed in (or opposite to) the neutrino spin direction. In Fig. 4 we show the resulting asymmetry

$$
A=\frac{N^{+}-N^{-}}{N^{+}+N^{-}}
$$

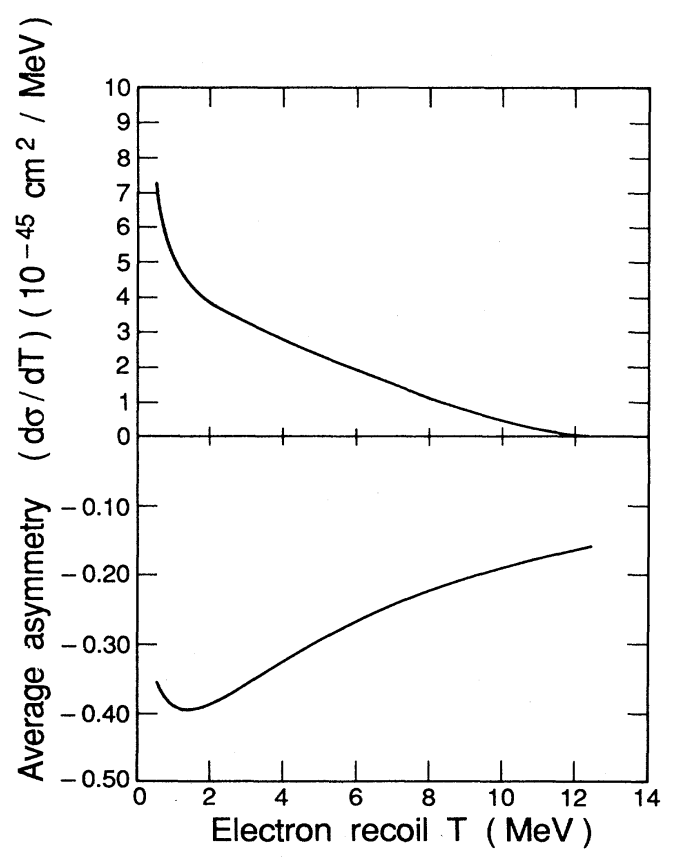

FIG. 4. Upper part: cross section for $v_{e}$ scattering on electrons, averaged over the $\beta$-decay spectrum of ${ }^{8} \mathbf{B}$. A neutrino magnetic moment $\mu_{v}=10^{-10}$ is assumed. Lower part: average azimuthal asymmetry with respect to the plane normal to the transverse neutrino polarization vector. where $N^{ \pm}$is the number of counts in the \pm hemisphere defined by the plane perpendicular to the neutrino transverse spin direction. The upper part of the figure shows the differential cross section averaged over the ${ }^{8} \mathrm{~B}$ spectrum. In calculating it we took into account the $\frac{1}{3}$ reduction of the weak interaction and assumed a magnetic moment $\mu_{v_{e}}=10^{-10}$ The asymmetry is sizable, at least for the parameters we chose. Its observation would be difficult, however, because the recoil electrons are strongly forward peaked; 5-MeV electrons, for instance, emerge at an angle of only about $12^{\circ}$ from the incoming neutrino direction. We note as an example of current technology that the proposed Sudbury heavy-water experiment ${ }^{20}$ will have an angular resolution of about $25^{\circ}$, placing detection of the asymmetry out of reach for the time being. If experimentally possible, though, observation of the phenomenon would constitute convincing proof of the mechanism proposed by Voloshin, Vysotskii, and Okun. ${ }^{2}$

\section{REMARKS ON THE NEUTRINO CHARGE RADIUS}

The purely electromagnetic interactions of a neutral particle such as the neutrino can be described at lowmomentum transfer by two phenomenological parameters, the anomalous magnetic moment discussed above, and the mean-square charge radius $\left\langle r^{2}\right\rangle$. The latter conserves helicity in collisions with charged particles, and as a result has the same effect on the neutrino as a renormalization of the vector coupling constant $g_{V}$. From (A5) it follows that there is no particular advantage to lowenergy neutrinos in experiments to determine $\left\langle r^{2}\right\rangle$. Data on high-energy $v_{\mu}$-electron scattering ${ }^{13}$ can be interpreted as limiting positive $\left\langle r_{v_{\mu}}^{2}\right\rangle$ to $\leq 0.81 \times 10^{-32} \mathrm{~cm}^{2}$ and negative $\left\langle r_{\mu_{v}}^{2}\right\rangle$ to $\geq-7.3 \times 10^{-32} \mathrm{~cm}^{2}$.

A parametrization of the electromagnetic current solely in terms of $\mu$ and $\left\langle r^{2}\right\rangle$ ignores the weak effects that, within the standard model, are intrinsically linked to the electromagnetic interaction. Formula (A5), therefore, will govern the behavior of composite neutrinos, but does not strictly describe all the radiative corrections generated by the standard model. There, the neutrino charge radius arises in the one-loop approximation not only from "proper" vertex diagrams but also from the $\gamma-Z$ selfenergy diagrams; with the usual definition of $\left\langle r^{2}\right\rangle$ [see Eq. (A2)] the charge radius is infinite and gauge dependent. ${ }^{21,22}$ This difficulty is overcome by direct consideration of radiative corrections to neutrino-lepton scattering $v_{l} l^{\prime} \rightarrow v_{l} l^{\prime}$. The scattering contains ${ }^{22}$ a form factor $F_{\gamma}\left(q^{2}\right)$ that enters the amplitude as

$$
M \propto \frac{F_{\gamma}\left(q^{2}\right)}{q^{2}} l_{\mu}^{\gamma},
$$

where $l_{\mu}^{\gamma}$ is the charged-lepton vector current. The appropriate derivative of this form factor yields the gaugeindependent finite neutrino electroweak radius, independent of the flavor of the charged lepton $l$ :

$$
\left\langle r_{v_{l}}^{2}\right\rangle_{E W}=\left.6 \frac{\partial F_{\gamma}\left(q^{2}\right)}{\partial q^{2}}\right|_{q^{2}=0} \approx \frac{G_{F}}{2 \sqrt{2} \pi^{2}} \ln \frac{M_{W}^{2}}{m_{l}^{2}}, \quad l=e, \mu, \tau,
$$


which has a value $3.2 \times 10^{-33} \mathrm{~cm}^{2}$ for the electron neutrino and $1.7 \times 10^{-33} \mathrm{~cm}^{2}$ for the muon neutrino. Presently existing experimental limits are only about 1 order of magnitude larger than the sensitivity required to actually see the effects of the radiative corrections ${ }^{21}$ within the standard model [not only those associated with the charge radius (6) but all radiative corrections taken together ${ }^{21}$ ], in contrast with the situation with the magnetic moment.

\section{CONCLUSIONS}

We have shown here that certain feasible laboratory experiments with low-energy neutrinos would be sensitive to magnetic moments of magnitude needed to explain the solar-neutrino deficit. Although other arguments indicate that this explanation is unlikely, a direct experiment is still worthwhile. If, by chance, neutrinos do have large magnetic moments, physics well beyond the standard model will be opened up.

\section{ACKNOWLEDGMENTS}

One of the authors (P.V.) acknowledges the hospitality of the Aspen Center for Physics, where this work began, and discussions with Professor S. Bludman and Professor A. Halprin. The work was supported by the U.S. Department of Energy under Contract No. DE-F603-88ER40397.

\section{APPENDIX: CROSS-SECTION FORMULAS}

Here we collect the cross-section formulas for scattering of neutrinos on electrons, nucleons, and nuclei. Many of them have been derived previously, but are scattered throughout the literature. In the following we assume that an ultrarelativistic Dirac neutrino is characterized by two phenomenological parameters, a magnetic moment $\mu_{v}$ expressed in units of the electron Bohr magnetons, and a charge radius $\left\langle r^{2}\right\rangle$. The neutrino electromagnetic vertex is then

$$
\Gamma^{\mu}=F_{1} \gamma^{\mu}-\frac{F_{2}}{2 m_{v}} \sigma^{\mu v} q_{v},
$$

where $q$ is the momentum transfer and

$$
F_{1}=\frac{1}{6} q^{2}\left\langle r^{2}\right\rangle, \quad F_{2}=\mu_{v} \frac{m_{v}}{m_{e}} .
$$

If a neutrino of energy $E_{v}$ scatters on a particle of mass $M$ at rest in the laboratory frame, two relevant observables are the recoil kinetic energy $T$ and the recoil angle $\chi$ (with respect to the neutrino beam). They are related by

$$
\cos \chi=\frac{E_{v}+M}{E_{v}}\left[\frac{T}{T+2 M}\right)^{1 / 2},
$$

and $T$ is restricted to values

$$
T \leq \frac{2 E_{v}^{2}}{2 E_{v}+M},
$$

We shall consider the differential cross sections in terms of the recoil kinetic energy $T$. Target particles can be electrons with electromagnetic form factors $F_{1}=1$, $F_{2}=0$, or nucleons (or nuclei) with electromagnetic form factors $F_{1}=Z$ (charge number),$F_{2}=\mu_{N}$ (anomalous magnetic moment in nuclear magnetons). Neutrinos exchange $Z$ particles with these targets as well, and electron neutrinos in particular also exchange $W$ 's. From (A1) it is apparent that the effect of the charge radius is coherent with those of the weak interactions (no helicity flip), while the effect of the magnetic moment is incoherent (helicity flip).

For neutrino-electron scattering we obtain

$$
\begin{aligned}
\frac{d \sigma}{d T}= & \frac{G_{F}^{2} m_{e}}{2 \pi}\left[\left(g_{V}+x+g_{A}\right)^{2}+\left(g_{V}+x-g_{A}\right)^{2}\left(1-\frac{T}{E_{v}}\right]^{2}\right. \\
& \left.+\left[g_{A}^{2}-\left(g_{V}+x\right)^{2}\right] \frac{m_{e} T}{E_{v}^{2}}\right] \\
& +\frac{\pi \alpha^{2} \mu_{v}^{2}}{m_{e}^{2}} \frac{1-T / E_{v}}{T},
\end{aligned}
$$

where

$$
\begin{aligned}
& g_{V}=\left\{\begin{array}{l}
2 \sin ^{2} \theta_{W}+\frac{1}{2} \text { for } v_{e}, \\
2 \sin ^{2} \theta_{W}-\frac{1}{2} \text { for } v_{\mu}, v_{\tau},
\end{array}\right. \\
& g_{A}=\left\{\begin{array}{l}
\frac{1}{2} \text { for } v_{e}, \\
-\frac{1}{2} \text { for } v_{\mu}, v_{\tau},
\end{array}\right.
\end{aligned}
$$

and

$$
x=\frac{\sqrt{2} \pi \alpha\left\langle r^{2}\right\rangle}{3 G_{F}}=\frac{2 M_{W}^{2}}{3}\left\langle r^{2}\right\rangle \sin ^{2} \theta_{W} .
$$

When antineutrinos are involved, we must make the substitution $g_{A} \rightarrow-g_{A}$.

For neutrino and antineutrino scattering on nucleons and on odd $A$ nuclei we shall consider only the helicity changing part of the cross section, i.e., the part containing $\mu_{v}^{2}$ :

$$
\begin{aligned}
\frac{d \sigma}{d T}=\frac{\pi \alpha^{2} \mu_{v}^{2}}{m_{e}^{2}}[ & \frac{1-T / E_{v}}{T} Z^{2}-\frac{T}{2 E_{v}^{2}} \mu_{N} Z \\
& \left.+\frac{\left(2-T / E_{v}\right)^{2}-2 M T / E_{v}^{2}}{8 M} \mu_{N}^{2}\right] .
\end{aligned}
$$

The characteristic $\sim 1 / T$ singularity is absent from the second and third terms of (A6), which correspond to magnetic dipole on magnetic dipole scattering. The cross section is dominated by the first term, describing magnetic dipole scattering in the Coulomb field; the $Z^{2}$ dependence of that term signifies coherence. ${ }^{12}$ For applications at neutrino energies of astrophysical significance the terms in (A6) containing the $\mu_{N}$ contribute negligibly.

For scattering of neutrinos on spin-zero nuclei of charge $Z_{e}$, the $\mu_{v}^{2}$-dependent cross section is 


$$
\frac{d \sigma}{d T}=\frac{\pi \alpha^{2} \mu_{v}^{2}}{m_{e}^{2}}\left[\frac{1-T / E_{v}}{T}+\frac{T}{4 E_{v}^{2}}\right] Z^{2} .
$$

Total cross sections are obtained from (A5)-(A7) by integrating over $T$ up to the maximum recoil given by (A4). The minimum recoil $T_{\min }$ is related to the distance over which the electron, nucleon, or nuclear charge is screened (or to the properties of the detection apparatus). The leading factor in the total cross sections for electrons (nucleons or nuclei) is

$$
\sim \ln \frac{2 E_{v}^{2}}{T_{\min }\left(2 E_{v}+m_{e}\right)} \quad\left(\sim \ln \frac{2 E_{v}^{2}}{T_{\min } M}\right) .
$$

Note that the term inside the logarithm is largest for electrons. In astrophysical applications it is important to remember the suppression of scattering on electrons by Fermi gas degeneracy, and the $Z^{2}$ coherence enhancement of scattering on nuclei.
Formula (A5) describes the scattering of the longitudinally polarized neutrinos on unpolarized electrons. The recoiling electrons are obviously distributed symmetrically with respect to the beam direction. However, when neutrinos have a nonvanishing magnetic moment they can be transversely polarized, e.g., by precession in the solar magnetic field. As pointed out in Ref. 19, the weak-electromagnetic interference will then induce an azimuthal angle dependence of the form (we show only the $\phi$-dependent part)

$$
\begin{aligned}
\frac{d \sigma}{d T d \phi}= & \frac{\alpha G_{F} \mu_{v}}{2 \sqrt{2} \pi m_{e}}\left[\left(g_{V}+g_{A}\right) \frac{T}{E_{v}}-2 g_{V}\right] \\
& \times\left(1+\frac{2 m_{e}}{T}\right)^{1 / 2}\left|\xi_{\perp}\right| \cos \phi,
\end{aligned}
$$

where $\xi_{\perp}$ is the transverse component of the neutrino polarization vector and $\cos \phi \equiv \hat{\boldsymbol{\xi}}_{\perp} \cdot \hat{\mathbf{p}}_{f}^{(e)}$.
${ }^{1}$ R. Davis, Jr., in Seventh Workshop on Grand Unification, proceedings, Toyama, Japan, 1986, edited by J. Arafune (World Scientific, Singapore, 1986).

${ }^{2}$ M. B. Voloshin, M. I. Vysotskii, and L. B. Okun, Zh. Eksp. Teor. Fiz. 91, 754 (1986) [Sov. Phys. JETP 64, 446 (1986)].

${ }^{3}$ B. W. Lee and R. E. Schrock, Phys. Rev. D 16, 1444 (1977); W. Marciano and A. I. Sanda, Phys. Lett. 67B, 303 (1977).

${ }^{4}$ M. A. B. Beg, W. J. Marciano, and M. Ruderman, Phys. Rev. D 17, 1395 (1977).

${ }^{5}$ M. Fukugita and T. Yanagida, Phys. Rev. Lett. 58, 1807 (1987); M. B. Voloshin, Report No., ITEP 215, 1988 (unpublished).

${ }^{6}$ G. G. Raffelt, Phys. Rev. D 39, 2066 (1989).

${ }^{7}$ B. Kayser, Phys. Rev. D 26, 1662 (1982).

${ }^{8}$ J. Morgan, Phys. Lett. 102B, 247 (1981); M. Fukugita and S. Yazaki, Phys. Rev. D 37, 3817 (1987).

${ }^{9}$ J. Bernstein et al., Phys. Rev. 132, 1227 (1963); P. Sutherland et al., Phys. Rev. D 13, 2700 (1976).

10J. M. Lattimer and J. Cooperstein, Phys. Rev. Lett. 61, 23 (1988).

${ }^{11}$ R. Barbieri and R. N. Mohapatra, Phys. Rev. Lett. 61, 27 (1988).

${ }^{12}$ D. Notzold, Phys. Rev. D 38, 1658 (1988).

${ }^{13}$ K. Abe et al., Phys. Rev. Lett. 58, 636 (1987); J. Dorenbosch et al., Z. Phys. 41, 567 (1989).

${ }^{14}$ R. C. Allen et al., Phys. Rev. Lett. 55, 2401 (1985).

${ }^{15}$ F. Reines, H. S. Gurr, and H. W. Sobel, Phys. Rev. Lett. 37, 315 (1976).

${ }^{16}$ A. V. Kyudliev, Nucl. Phys. B243, 387 (1984).

${ }^{17}$ F. Boehm and P. Vogel, Physics of Massive Neutrinos (Cambridge University Press, Cambridge, England, 1987).

${ }^{18}$ T. Kirsten, in Masive Neutrinos in Particle Physics and Astrophysics, proceedings of the Twenty-First Rencontre de Moriond (Sixth Workshop), Tignes, Savoie, France, 1986, edited by $O$. Fackler and J. Tran Thanh Van (Editions Frontiéres, Gif-sur-Yvette, 1986), p. 119.

${ }^{19}$ R. Barbieri and G. Fiorentini, in Neutrino Masses and Neutrino Astrophysics, proceedings of the Fourth Telemark Conference, Ashland, Wisconsin, 1987, edited by V. Barger et al. (World Scientific, Singapore, 1987).

${ }^{20} \mathrm{G}$. T. Ewan et al., Sudbury Neutrino Observatory Report No. SNO-87-12, 1987 (unpublished).

${ }^{21}$ W. J. Marciano and A. Sirlin, Phys. Rev. D 22, 2695 (1980); S. Sarantakos, A. Sirlin, and W. J. Marciano, Nucl. Phys. B217, 84 (1983).

22J. L. Lucio, A. Rosado, and A. Zepeda, Phys. Rev. D 31, 1091 (1985). 